Article

\title{
Otoprotective Effects of Zingerone on Cisplatin-Induced Ototoxicity
}

\author{
Chang Ho Lee, Da-hye Lee, So Min Lee and So Young Kim * \\ Department of Otorhinolaryngology, CHA University College of Medicine, Seongnam 13496, Korea; \\ hearwell@gmail.com (C.H.L.); ldada72@naver.com (D.-h.L.); lws6812@naver.com (S.M.L.) \\ * Correspondence: sossi81@hanmail.net; Tel.: +82-31-870-5340; Fax: +82-31-870-5346
}

Received: 19 March 2020; Accepted: 14 May 2020; Published: 15 May 2020

\begin{abstract}
Previous studies have described the effects of zingerone (ZO) on cisplatin (CXP)-induced injury to the kidneys, liver, and other organs but not to the cochlea. This study aimed to investigate the effects of ZO on CXP-induced ototoxicity. Eight-week-old Sprague-Dawley rats were used and divided into a control group, a CXP group, and a CXP + ZO group. Rats in the CXP group received $5 \mathrm{mg} / \mathrm{kg} /$ day CXP intraperitoneally for five days. Rats in the CXP $+Z O$ group received $5 \mathrm{mg} / \mathrm{kg} /$ day CXP intraperitoneally for five days and $50 \mathrm{mg} / \mathrm{kg} /$ day ZO intraperitoneally for seven days. Auditory brainstem response thresholds (ABRTs) were measured before (day 0) and after (day 10) drug administration. Cochlear histology was examined using hematoxylin and eosin (H\&E) staining and cochlear whole mounts. The expression levels of cytochrome P450 (CYP)1A1, CYP1B1, inducible nitric oxide synthase ( $i N O S)$, nuclear factor kappa B (NFKB), tumor necrosis factor alpha (TNF $\alpha$ ), and interleukin 6 (IL6) were estimated using quantitative reverse transcription-polymerase chain reaction. The expression levels of heme oxygenase 1 (HO1) and caspase 3 were analyzed via Western blotting. The auditory thresholds at 4,8 , and $16 \mathrm{kHz}$ were attenuated in the CXP $+\mathrm{ZO}$ group compared with the CXP group. The mRNA expression levels of CYP1A1, CYP1B1, iNOS, NFKB, $T N F \alpha$, and IL6 were lower in the CXP + ZO group than in the CXP group. The protein expression levels of $\mathrm{HO} 1$ and caspase 3 were lower in the CXP + ZO group than in the CXP group. Cotreatment with $\mathrm{ZO}$ exerted otoprotective effects against $C X P$-induced cochlear injury via antioxidative and anti-inflammatory activities involving $C Y P S$, $i N O S, N F \kappa B$, and $T N F \alpha$.
\end{abstract}

Keywords: hearing loss; cisplatin; zingerone; cytochrome P450; tumor necrosis factor alpha

\section{Introduction}

Oxidative stress combined with inflammation in the cochlea is one of the main pathophysiologies of cochlear hearing loss [1-3]. Noise-induced hearing loss, age-related hearing loss, and hearing loss induced by ototoxic drugs, including aminoglycosides and anticancer agents, have been reported to be mediated by oxidative stress and inflammation in cochlear hair cells and spiral ganglion cells [1-3]. Cisplatin (CXP)-induced ototoxicity is accompanied by increases in the levels of reactive oxygen species; the inflammatory molecules tumor necrosis factor alpha (TNF $\alpha$ ), interleukin $1 \beta$ (IL1 $\beta$ ), and interleukin 6 (IL6);

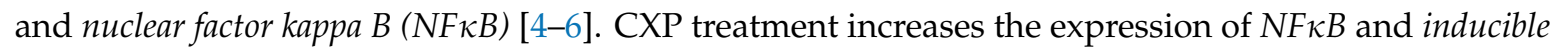
nitric oxide synthase (iNOS) in the cochlea, especially in the stria vascularis and spiral ligament [4]. Activation of $N F \kappa B$ increases the levels of proinflammatory cytokines, such as TNF $\alpha$, IL1 $\beta$, and IL6, in the cochlea [7]. These oxidative stress responses and inflammatory responses induce apoptosis activation by activating caspase 3 [8]. The sensory hair cells of the organ of Corti, the stria vascularis, and spiral ganglion cells have been reported to be the main targets of CXP-induced oxidative and inflammatory damage $[9,10]$. Animal studies have shown that these ototoxic injuries are initiated by the degeneration and loss of cochlear outer hair cells and are accompanied by neuronal death and spiral 
ganglion cell degeneration. This histological damage is associated with shifts in the auditory brainstem response (ABR) threshold (ABRT) and optoacoustic emissions, which have been characterized as dose-dependent, and with irreversible hearing loss [10,11]. A number of in vivo and in vitro studies have suggested treatment with preservative agents to limit CXP-induced ototoxic injury [8,12-14]. However, the clinical use of these agents has been impeded by the questionable and potentially toxic effects of human doses.

Zingerone (ZO; 4-para methoxy-4-hydroxyphenyl-2-butanone) has antioxidant, anti-inflammatory, and antiapoptotic effects [15] that are presumed to be related to its protective effects against radiationinduced intestinal injury [16], lipopolysaccharide-induced liver damage [17], and sepsis-triggered nephrotoxicity [18]. Moreover, ZO has protective effects against CXP-induced toxicities, such as nephrotoxicity [15,19], hepatotoxicity [20], cardiotoxicity [21], and ovarian and uterine toxicity [22]. Because the molecular mechanisms underlying CXP-induced nephrotoxicity overlap with those underlying CXP-induced ototoxicity [23], it would be reasonable to expect that $\mathrm{ZO}$ has otoprotective effects against CXP-induced ototoxicity. However, to the best of our knowledge, no study has investigated the protective effect of $\mathrm{ZO}$ against ototoxicity.

Here, we hypothesized that the antioxidative, anti-inflammatory, and antiapoptotic effects of ZO might be effective against CXP-induced ototoxicity. To test this hypothesis, adult rats were cotreated with ZO during exposure to CXP. We used the dose of ZO reported in previous studies on nephrotoxicity [19]. We simultaneously pretreated rats with both ZO and CXP instead of with only $\mathrm{ZO}$, as was done in previous studies [19]. The results demonstrated preservation of auditory function and cochlear histology in cochlear outer hair cells and spiral ganglion cells. In addition, the increases in the expression levels of $i N O S, N F \kappa B$, the proinflammatory molecules $T N F \alpha$ and $I L 6$, and caspase 3 were reduced in the ZO-treated group. The present results broaden the application of $\mathrm{ZO}$ to ototoxicity and suggest a new approach to limit CXP-induced ototoxic injury.

\section{Results}

Auditory thresholds were increased after CXP injection (Figure 1). The changes in auditory thresholds differed significantly among the groups $(p<0.001$ for both pretreatment and post-treatment and for frequencies of 4, 8, 16, and $32 \mathrm{kHz}$; repeated measures ANOVA). The auditory threshold was higher in the CXP group than in the control group on day $10(p<0.001$, repeated measures ANOVA with Tukey's test). The mean auditory thresholds in the CXP group on day 10 were $51.88(\mathrm{SD}=3.44)$ decibel sound pressure level (dB SPL), $61.88(\mathrm{SD}=5.26) \mathrm{dB}$ SPL, $58.75(\mathrm{SD}=3.40) \mathrm{dB}$ SPL, and 58.75 $(\mathrm{SD}=3.75) \mathrm{dB}$ SPL for $4,8,16$, and $32 \mathrm{kHz}$, respectively. The auditory thresholds in the CXP $+\mathrm{ZO}$ group were lower than those in the CXP group on day $10(p=0.001$, repeated measures ANOVA with Tukey's test). The mean auditory thresholds in the CXP + ZO group on day 10 were $37.5(\mathrm{SD}=2.5)$ dB SPL, 37.5 (SD = 2.81) dB SPL, $43.13(\mathrm{SD}=3.84) \mathrm{dB}$ SPL, and $50.00(\mathrm{SD}=3.03) \mathrm{dB}$ SPL for 4, 8, 16, and $32 \mathrm{kHz}$, respectively.

The cochlear mRNA expression levels of cytochrome P450 (CYP)1A1, CYP1B1, iNOS, NFkB, IL6, and TNF $\alpha$ were higher in the CXP group than in the control group, and these increases were reversed in the CXP + ZO group (Figure 2). The CYP1A1 mRNA levels in the CXP and CXP + ZO groups were 3.59-fold $(\mathrm{SD}=0.90)$ and 0.81-fold $(\mathrm{SD}=0.13)$ higher, respectively, than the level in the control group ( $p=0.003$ with ANOVA, $p=0.008$ with Tukey's test for control vs. CXP, and $p=0.005$ with Tukey's test for CXP vs. CXP + ZO). The CYP1B1 mRNA levels in the CXP and CXP + ZO groups were 5.93-fold $(\mathrm{SD}=1.18)$ and 1.35 -fold $(\mathrm{SD}=0.11)$ higher, respectively, than the level in the control group $(p<0.001$ with ANOVA, $p<0.001$ with Tukey's test for control vs. CXP, and $p<0.001$ with Tukey's test for CXP vs. $\mathrm{CXP}+\mathrm{ZO})$. The $i N O S$ mRNA levels in the CXP and CXP + ZO groups were 4.16-fold (SD =1.19) and 1.59-fold $(\mathrm{SD}=0.18)$ higher, respectively, than the level in the control group $(p=0.011$ with ANOVA, $p=0.012$ with Tukey's test for control vs. CXP, and $p=0.045$ with Tukey's test for CXP vs. CXP + ZO). The NF $K B$ mRNA levels in the CXP and CXP + ZO groups were 5.13-fold (SD = 1.11) and 2.56-fold $(\mathrm{SD}=0.73)$ higher, respectively, than the level in the control group $(p=0.004$ with ANOVA, $p=0.003$ 
with Tukey's test for control vs. CXP, and $p=0.070$ for CXP vs. CXP + ZO). The IL6 mRNA levels in the CXP and CXP + ZO groups were 6.40-fold (SD = 1.21) and 1.47-fold (SD =0.30) higher, respectively, than the level in the control group ( $p<0.001$ with ANOVA, $p<0.001$ with Tukey's test for control vs. CXP and CXP vs. CXP + ZO). The TNF $\alpha$ mRNA levels in the CXP and CXP + ZO groups were 5.59-fold ( $\mathrm{SD}=1.05)$ and 1.39-fold $(\mathrm{SD}=0.02)$ higher, respectively, than the level in the control group $(p<0.001$ with ANOVA, $p<0.001$ with Tukey's test for control vs. CXP and CXP vs. CXP + ZO).
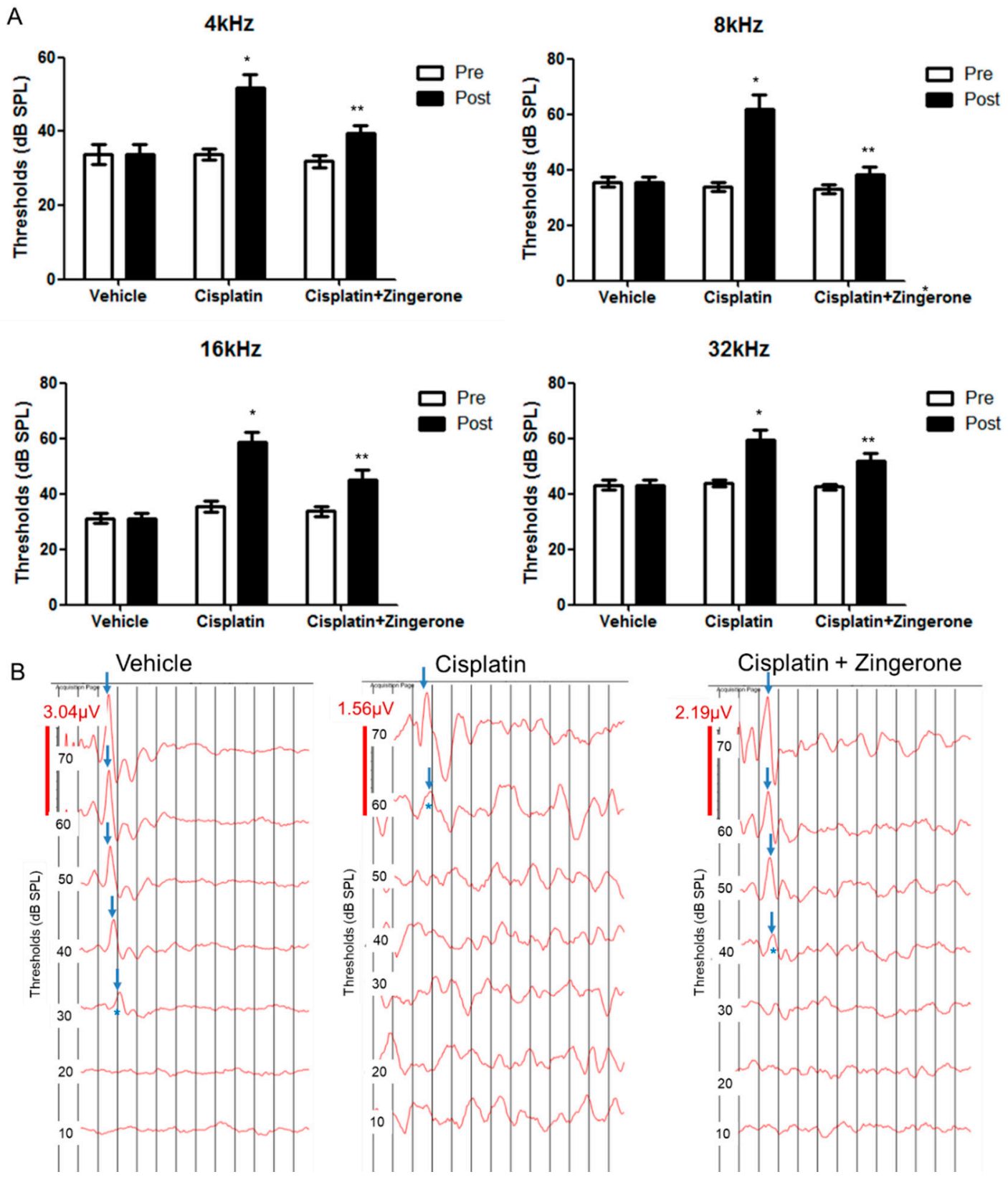

Figure 1. Auditory brainstem response (ABR) thresholds on day 0 (pretreatment) and day 10 (post-treatment). (A) The ABR thresholds differed among the three groups $\left({ }^{*} p<0.05\right.$ for the control vs. cisplatin groups by repeated measures ANOVA with Tukey's posthoc test). The ABR thresholds in the cisplatin + zingerone group on day 10 were attenuated compared with those in the cisplatin group ${ }^{* *} p<0.05$ for the cisplatin vs. cisplatin + zingerone groups by repeated measures ANOVA with Tukey's posthoc test). The values shown in the graphs are the means \pm standard deviations. (B) The ABR waveforms at $8 \mathrm{kHz}$ are presented for each group (the arrows indicate wave II; ${ }^{*}$ indicates ABR thresholds). 

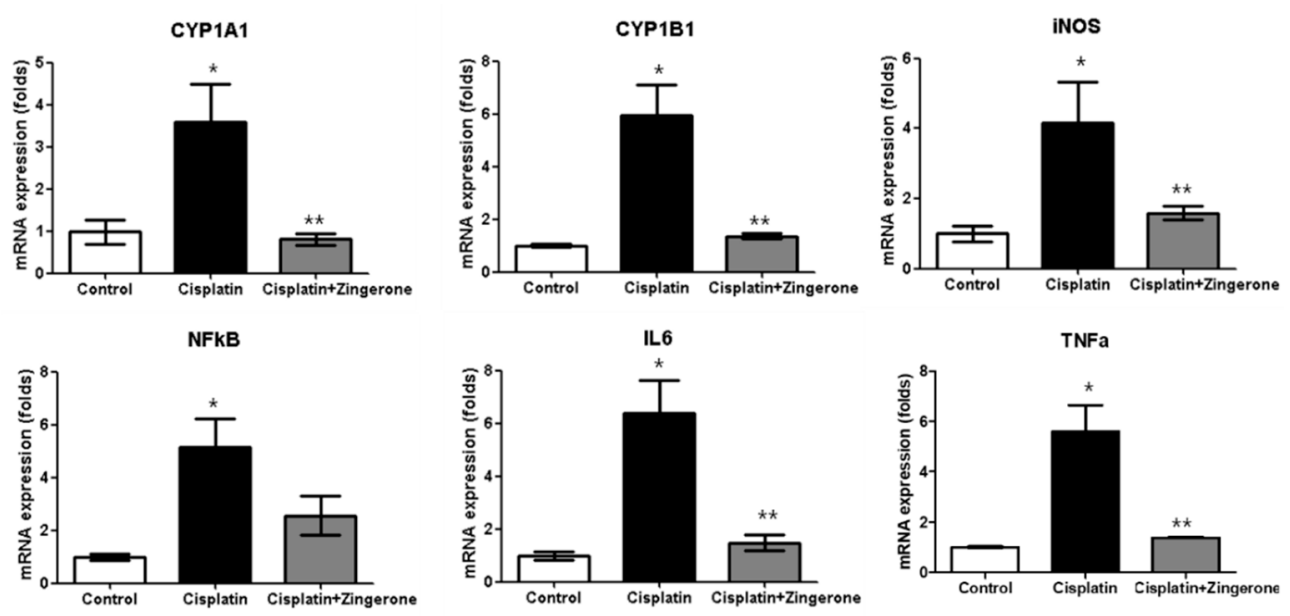

Figure 2. The mRNA expression levels of cytochrome P450 (CYP)1A1, CYP1B1, inducible nitric oxide synthase (iNOS), nuclear factor- $\mathrm{KB}(\mathrm{NF} \mathrm{B}$ ), tumor necrosis factor alpha (TNF $\alpha$ ), and interleukin 6 (IL6) were higher in the cisplatin (CXP) group than in the control group $\left({ }^{*} p<0.05\right.$, ANOVA with Tukey's posthoc test). The cisplatin + zingerone group exhibited lower mRNA expression levels of CYP1A1, CYP1B1, iNOS, NFkB, TNF $\alpha$, and IL6 than the cisplatin group (** $p<0.05$, ANOVA with Tukey's posthoc test). The values shown in the graphs are the means \pm standard deviations.

The cochlear protein expression levels of the aryl hydrocarbon receptor (AhR), heme oxygenase 1 (HO1), superoxide dismutase 2 (SOD2), and caspase 3 were higher in the CXP group than in the control group (Figure 3). In the CXP $+\mathrm{ZO}$ group, the increase in the expression of AhR and caspase 3 was smaller than that in the CXP group. The AhR protein level was lower in the CXP + ZO group than in the CXP group, but the levels in these groups were 1.44-fold $(\mathrm{SD}=0.04)$ and 1.66 -fold $(\mathrm{SD}=0.05)$ higher, respectively, than the level in the control group ( $p<0.001$ with ANOVA, $p<0.001$ with Tukey's test for control vs. CXP, and $p=0.07$ with Tukey's test for CXP vs. CXP $+Z \mathrm{Z})$. The HO1 protein level did not differ significantly between the CXP and CXP + ZO groups, and the levels in these groups were 2.15 -fold $(\mathrm{SD}=0.40)$ and 1.77 -fold $(\mathrm{SD}=0.22)$ higher, respectively, than that in the control group $(p=0.038$ with ANOVA, $p=0.033$ with Tukey's test for control vs. CXP, and $p=0.18$ with Tukey's test for CXP vs. CXP + ZO). The SOD2 protein level did not differ significantly between the CXP and $\mathrm{CXP}+\mathrm{ZO}$ groups, and the levels in these groups were 1.51-fold $(\mathrm{SD}=0.05)$ and 1.40 -fold $(\mathrm{SD}=0.11)$ higher, respectively, than that in the control group $(p<0.001$ with ANOVA, $p<0.001$ with Tukey's test for control vs. CXP, and $p=0.206$ with Tukey's test for CXP vs. CXP $+\mathrm{ZO}$ ). The caspase 3 protein levels were 2.03-fold $(\mathrm{SD}=0.16)$ and 1.33-fold $(\mathrm{SD}=0.20)$ higher in the CXP and CXP + ZO groups, respectively, than in the control group ( $p<0.001$ with ANOVA, $p<0.001$ with Tukey's test for control vs. CXP, and $p=0.115$ with Tukey's test for CXP vs. CXP + ZO).

Cochlear hematoxylin and eosin (H\&E) staining revealed a higher density of spiral ganglion cells in the CXP $+\mathrm{ZO}$ group than in the CXP group (Figure 4). Compared with the spiral ganglion cells in the CXP $+\mathrm{ZO}$ group, those in the CXP group contained vacuoles with condensed chromatin and were disordered. Cochlear whole mount staining ( $n=2$ per group, for a total of 6 cochleae) demonstrated the loss of outer hair cells in the CXP group, while these cells were preserved in the CXP + ZO group. The loss of outer hair cells was most commonly observed in the basal turn of the cochlea (percentage of outer hair cell survival (mean of both cochleae) $=98.6 \%(122 / 124)$ in the control group, $82.25 \%(102 / 124)$ in the CXP group, and 90.3\% (112/124) in the CXP + ZO group). 

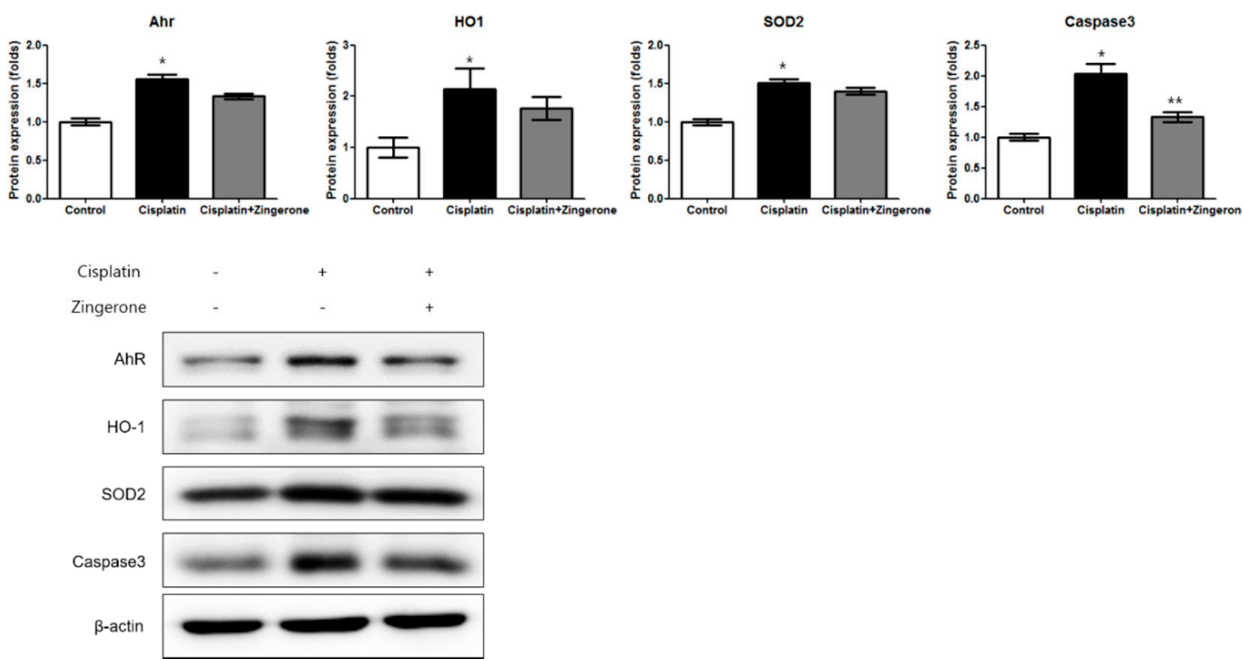

Figure 3. The protein expression levels of the aryl hydrocarbon receptor (AhR), heme oxygenase 1 (HO1), superoxide dismutase 2 (SOD2), and caspase 3 were higher in the cisplatin group than in the control group ( ${ }^{*} p<0.05$, ANOVA with Tukey's post hoc test, control vs. cisplatin groups; ${ }^{* *} p<0.05$, ANOVA with Tukey's post hoc test, cisplatin vs. cisplatin + zingerone groups). The protein expression levels of caspase 3 were lower in the cisplatin + zingerone group than in the cisplatin group $(p=0.03$, ANOVA with Tukey's posthoc test). The values shown in the graphs are the means \pm standard deviations. (+: not administered, -: administered).
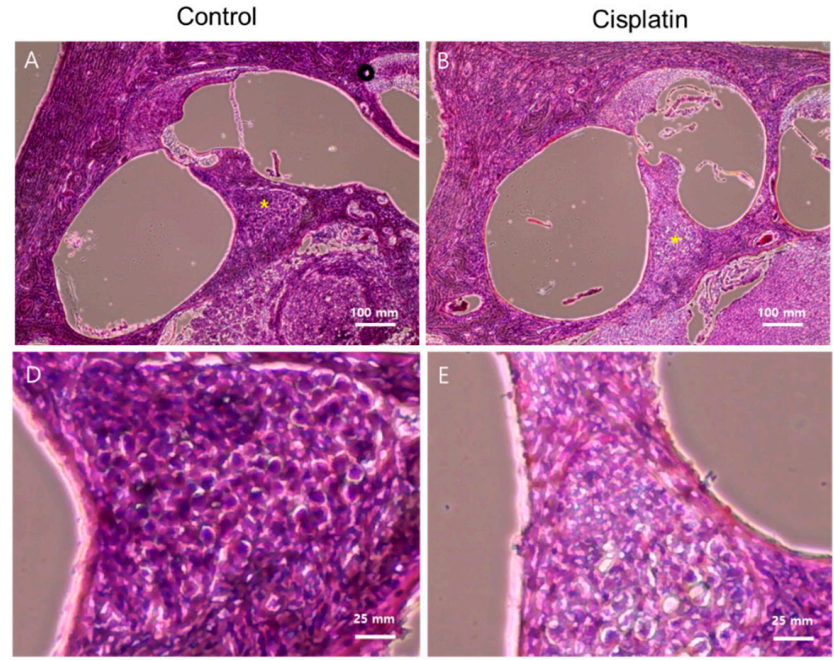

Cisplatin + Zingerone
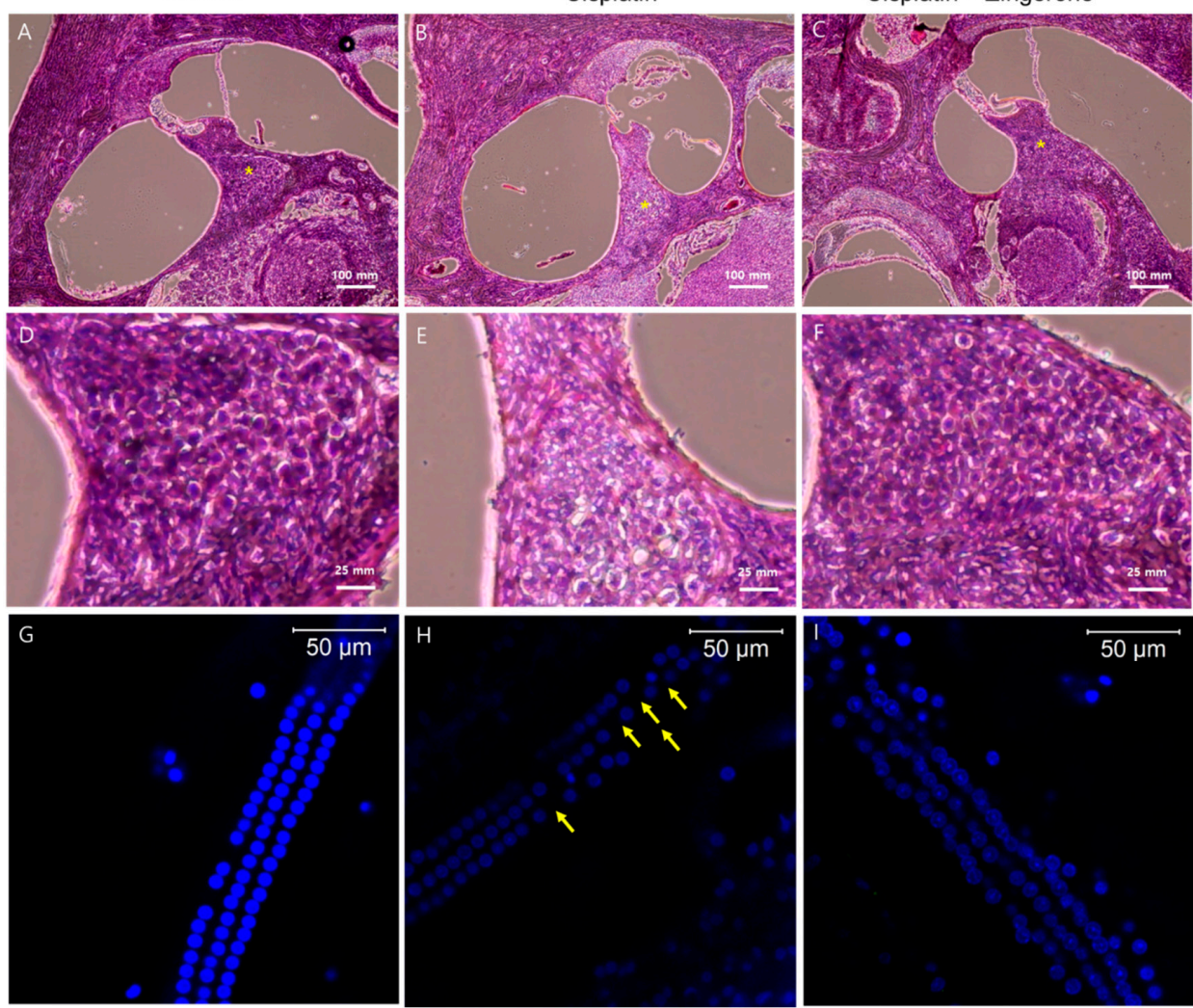

Figure 4. Hematoxylin and eosin (H\&E) staining of the cochlea (A-F) and whole mounts of the cochlear basal turn (G-I). The control group exhibited intact spiral ganglion cells (A) and outer hair cells $(\mathbf{G})$. Compared with the cisplatin group $(\mathbf{B}, \mathbf{E}, \mathbf{H})$, the cisplatin + zingerone group $(\mathbf{C}, \mathbf{F}, \mathbf{I})$ exhibited a preserved number and arrangement of spiral ganglion cells ( ${ }^{*}$ in $\mathbf{A}-\mathbf{C}$ and $\mathbf{D}-\mathbf{F}$ ) and outer hair cells (the arrows indicate the loss of outer hair cells). 


\section{Discussion}

$\mathrm{ZO}$ attenuated CXP-induced ototoxicity in a rat model. The effects of $\mathrm{ZO}$ were assessed functionally via measurement of ABRT reduction and morphologically via analysis of cochlear outer hair cell and spiral ganglion cell counts and arrangements. ZO exerted its antioxidative effect by decreasing the expression of CYP1A1, CYP1B1, and $i N O S$. This alleviation of oxidative stress was linked with attenuated expression of $N F \kappa B$ and proinflammatory molecules (TNF $\alpha$ and IL6). In addition, the decreased apoptotic activity could be predicted by the decreased expression of caspase 3 . In summary, the antioxidative, anti-inflammatory, and antiapoptotic effects of ZO on CXP-induced injury occur in the cochlea as well as in the kidneys and other organs. Because ZO is an herbal compound that is used to prevent and treat other diseases and symptoms, it can be used in patients concerned about ototoxic injury resulting from anticancer treatment with CXP. The equivalent dose of ZO in humans is expected to be $3 \mathrm{~g}$ in adults $(50 \mathrm{mg} / \mathrm{kg} \times 60 \mathrm{~kg}$ ), which is a feasible dose without definite toxicity. Further study on the clinical application of $Z O$ for ototoxicity is warranted. To our knowledge, this report is the first to describe the effect of $\mathrm{ZO}$ on ototoxic injury.

The antioxidative and anti-inflammatory effects of $Z O$ have been described in two CXP-induced nephrotoxicity model studies [15,19]. Both studies used identical doses of ZO $(50 \mathrm{mg} / \mathrm{kg} / \mathrm{day}$, administered three times) and CXP $(7 \mathrm{mg} / \mathrm{kg}$, administered one time) $[15,19]$. In addition, previous studies have demonstrated the dose-dependent antioxidative and anti-inflammatory effects of $\mathrm{ZO}$ and the lack of adverse effects on body weight or kidney function with doses of 10, 20, and $50 \mathrm{mg} / \mathrm{kg}$ [19]. Therefore, the dose of ZO used in the present study $(50 \mathrm{mg} / \mathrm{kg} /$ day, administered three times) was identical to that used in those previous studies. However, the dose of CXP used in this study $(5 \mathrm{mg} / \mathrm{kg} /$ day, administered three times) was higher than that used in previous studies. Although a higher dose of CXP was used in the current study than in previous studies, the antioxidative and anti-inflammatory effects of $\mathrm{ZO}$ were observed in the cochlea.

ZO cotreatment attenuated CXP-induced expression of the CYP enzymes CYP1A1 and CYP1B1 in the cochlea. CYP enzymes belong to a superfamily of mono-oxygenases and are involved in oxidative stress responses after CXP-induced injury [24]. A previous CXP-induced hepatotoxicity study in rats also reported attenuation of CYP enzyme expression in the $\mathrm{ZO}$ pretreatment group [20]. Because $\mathrm{ZO}$ is a polyphenolic alkanone, the phenolic component might play a role as a free radical scavenger and exert cytoprotective effects [25]. In addition, the expression of $i N O S$ was reduced in the ZO cotreatment group in the present study. CXP-induced ototoxic injury has been reported to induce the expression of iNOS, a major enzyme that generates nitric oxide [26]. Similar to the present study, a few previous studies showed that after $\mathrm{ZO}$ treatment, a reduction in $i N O S$ expression occurred in other organs, such as the liver [20], ovaries, and uterus [22].

In addition to exerting antioxidative effects, $\mathrm{ZO}$ cotreatment reduced the inflammatory response involving $N F \kappa B, T N F \alpha$, and IL6 in the cochlea. $N F \kappa B$ participates in mitogen-activated protein kinase signaling cascades, which activate proinflammatory cytokines and chemokines [27]. NF $B$ activation induced by CXP-mediated injury increases the secretion of preformed proinflammatory cytokines, including TNF $\alpha$ and IL6, without increases in their mRNA or protein expression [7]. This immediate secretion of proinflammatory cytokines was found to occur within $24 \mathrm{~h}$ after CXP treatment in an in vitro study [7]. Preformed TNF $\alpha$ is reportedly stored in inflammatory cells, such as mast cells and neutrophils $[28,29]$. Because we cotreated rats with ZO and CXP, these preformed inflammatory cytokines might have been released and might have induced inflammation; this possibility was supported by the elevated expression levels of TNF $\alpha$ and IL6 observed in the CXP + ZO group compared with the control group. TNF $\alpha$ has been reported to play a pivotal role in initiating inflammatory responses subsequent to CXP ototoxicity [7]. Blockade of TNF $\alpha$ activation by the TNF $\alpha$ inhibitor etanercept suppresses the secretion of proinflammatory cytokines, including IL6, in the peripheral blood [7]. On the other hand, activation of $T N F \alpha$ can activate $N F \kappa B$ via phosphorylation of inhibitr of nuclear factor kappa B (IкB) kinase and intranuclear translocation of $N F \kappa B$ [30]. By inhibiting 
the de novo synthesis of TNF $\alpha$ and $N F \kappa B, Z \mathrm{Z}$ can terminate the vicious cycles of CXP-induced inflammatory responses.

In the present study, CXP-induced apoptosis related to caspase 3 was attenuated in the cochlea in the $\mathrm{ZO}$ cotreatment group, although the caspase 3 level in this group was still higher than that in the control group. The immediate inflammatory and apoptotic responses after CXP administration could not be reversed in this study because ZO and CXP were simultaneously administered; in contrast, in previous studies, ZO was administered before CXP was injected [21,22]. Moreover, although ZO showed protective effects against oxidative stress and inflammation, it could not prevent activation of other apoptotic pathways, such as the intrinsic pathway involving mitochondrial cytochrome $c$ and caspases 9, 6, and 7 [31] or activation of the transient receptor potential vanilloid 1 channel in cochlear hair cells [32]. The combination of another therapy that inhibits these other CXP-induced injuries with $\mathrm{ZO}$ might be a promising therapeutic option for CXP-induced ototoxicity.

\section{Materials and Methods}

\subsection{Animal Treatments}

Eight-week postnatal female Sprague-Dawley rats $(n=24)$ were used for experiments. The Institutional Animal Care and Use Committee of CHA University Medical School (IACUC190047, 7 December, 2018) approved the experiments. All experiments were conducted according to the institutional guidelines. Rats were classified into a control group $(n=8)$, CXP group $(n=8)$, or CXP + ZO group ( $n=8$, Figure 5). Rats in the CXP group were administered $5 \mathrm{mg} / \mathrm{kg} /$ day CXP intraperitoneally from days 1 to 5 . Rats in the CXP $+Z O$ group received $50 \mathrm{mg} / \mathrm{kg} /$ day $Z O$ intraperitoneally, immediately after CXP injection, from days 1 to 7 . The dose of $Z O$ was determined from previous studies $[19,22]$. No weight loss or death occurred after $\mathrm{ZO}$ administration in this study. Rats in the control group received an identical volume of normal saline by intraperitoneal injection from days 1 to 7 .

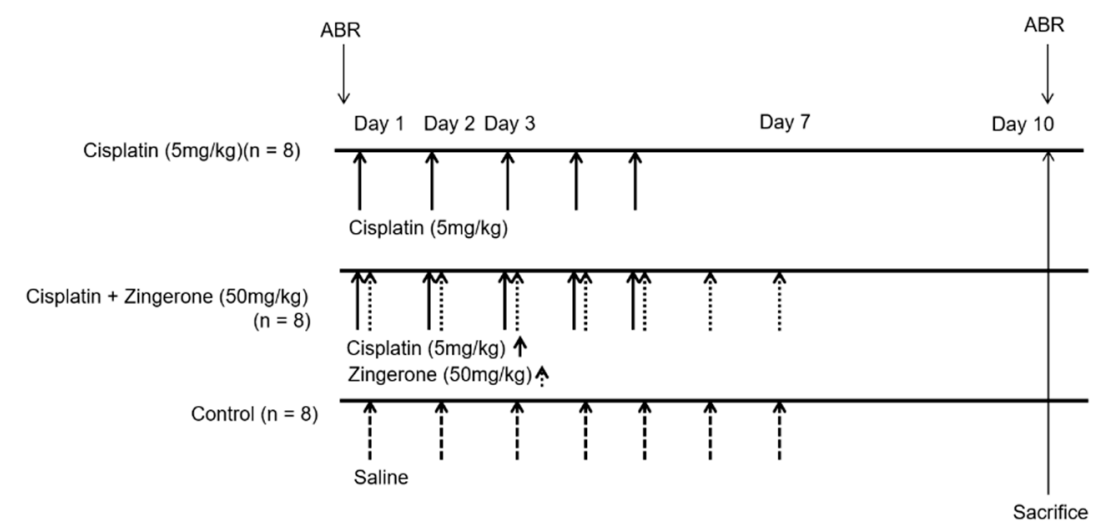

Figure 5. Dosing schedules and measurements used in the present study. Cisplatin $(5 \mathrm{mg} / \mathrm{kg}) \mathrm{was}$ administered to rats in the cisplatin group for 5 days $(n=10)$. Cisplatin $(5 \mathrm{mg} / \mathrm{kg})$ was administered for 5 days and zingerone $(50 \mathrm{mg} / \mathrm{kg}$ ) was administered for 7 days to rats in the cisplatin + zingerone group $(n=10)$. An equal volume of saline was administered to rats in the control group $(n=10)$. Auditory brainstem responses (ABRs) were measured on days 0 and 10.

The ABRTs for a tone burst at 4, 8, 16, or $32 \mathrm{kHz}$ were measured on days 0 and 10, as performed in a previous study [33]. Rats were anesthetized by intraperitoneal injection of a mixture of Zoletil $(40 \mathrm{mg} / \mathrm{kg})$ and xylazine $(10 \mathrm{mg} / \mathrm{kg})$, and electrodes were inserted at the vertex and the ipsilateral and contralateral retroauricular areas. The tone bursts (duration, $1562 \mu \mathrm{s}$; envelope, Blackman; stimulation rate, 21.1/s) were delivered via an EC1 (electrostatic speaker - coupler model). The auditory evoked responses with 1024 sweeps were averaged. The auditory threshold was defined as the lowest sound intensity that evoked wave II [34]. 
The experimenters were blinded to the treatments of the rats. All cochleae were dissected immediately after ABR measurement on day 10. After dissection of the bony labyrinth, membranous labyrinth tissues were harvested from 18 rats ( $n=6$ per group). A total of 18 cochleae from 9 rats ( $n=3$ per group) were analyzed with quantitative reverse transcription-polymerase chain reaction (RT-PCR), and another 18 cochleae were analyzed by Western blotting. The remaining 12 cochleae from 6 rats were immersion-fixed in a $\%$ paraformaldehyde solution for histological examination.

\subsection{Histological Examination}

For hematoxylin and eosin (H\&E) staining, paraffin blocks of decalcified cochleae were prepared as previously described [35]. Ten-micron-thick cochlear sections were sliced from the paraffin blocks using a rotary microtome. After mounting on glass slides, sections were deparaffinized, incubated in hematoxylin for $5 \mathrm{~min}$, and stained with eosin for $45 \mathrm{~s}$. Cochlear histological examinations were performed using an EVOS ${ }^{\mathrm{TM}}$ XL Core Imaging System (Invitrogen by Thermo Fisher Scientific, Waltham, MA, USA \#AMEX1000). For cochlear whole mounts, cochlear outer hair cells ( $n=2$ per group for a total of 6 cochlea) were dissected after decalcification. The dissected cochlear outer hair cells were stained with 4',6-diamidino-2-phenylindole (DAPI) as previously described and imaged using confocal microscopy [36].

\subsection{Quantitative RT-PCR Analysis}

Quantitative RT-PCR was conducted as previously described [37]. In brief, total RNA was extracted from cochleae using TRI Reagent ${ }^{\circledR}$ (Sigma-Aldrich, St. Louis, MO, USA). TOPscript ${ }^{\mathrm{TM}}$ RT DryMIX (dT18 plus; Enzynomics Co. Ltd., Daejeon, South Korea) was used for the reverse transcription reaction. Quantitative RT-PCR was performed with TOPreal ${ }^{\mathrm{TM}} \mathrm{qPCR} 2 \times$ PreMIX (SYBR Green with low ROX; Enzynomics, Daejeon, Korea) and oligonucleotide primers (Table 1) in a CFX96 Touch ${ }^{\text {TM }}$ Real-Time PCR Detection System (Bio-Rad, Hercules, CA, USA). mRNA expression levels were estimated based on the expression level of the reference gene glyceraldehyde 3-phosphate dehydrogenase (GAPDH).

Table 1. Oligonucleotide primer sequences used for quantitative reverse transcription-polymerase chain reaction.

\begin{tabular}{|c|c|c|c|c|c|}
\hline Gene & Primer Sequence (Forward) & Primer Sequence (Reverse) & $\begin{array}{l}\text { Annealing } \\
\text { Temperature } \\
\left({ }^{\circ} \mathrm{C}\right)\end{array}$ & $\begin{array}{l}\text { Product } \\
\text { Size (bp) }\end{array}$ & $\begin{array}{l}\text { RefSeq } \\
\text { Number }\end{array}$ \\
\hline CYP1A1 & 5'- CATCCCCCACAGCACCATAA - $3^{\prime}$ & 5'- TTCGCTTGCCCAAACCAAAG -3' & 60 & 212 & NM_012540.2 \\
\hline CYP1B1 & 5'- TGCTACTCGTTTCGGTCCTG -3' & 5'- CAAGGCGAGCGAAGTACAAG -3' & 60 & 162 & NM_012940.2 \\
\hline$i N O S$ & 5' - AGGCCACCTCGGATATCTCT -3' & 5' - TCTCTGGGTCCTCTGGTCAA -3' & 60 & 85 & NM_012611.3 \\
\hline$N F \kappa B$ & 5'- TGTCTGCACCTGTTCCAAAGA-3' & 5'- TGCCAGGTCTGTGAACACTC-3' & 60 & 143 & NM_199267.2 \\
\hline IL6 & 5'- AGAGACTTCCAGCCAGTTGC-3' & 5'- TGAAGTCTCCTCTCCGGACT-3' & 60 & 88 & NM_012589.2 \\
\hline$T N F \alpha$ & 5'- CGTCAGCCGATTTGCCATTT -3' & 5'- TCCCTCAGGGGTGTCCTTAG -3' & 60 & 88 & NM_012675.3 \\
\hline
\end{tabular}

\subsection{Western Blot Analysis}

Western blot analysis was conducted as previously described [37]. In brief, radioimmunoprecipitation assay buffer (Cell Signaling Technology, Danvers, MA, USA) was used to lyse cochleae, and the protein concentration was measured using a Bio-Rad Protein Assay Kit (Bio-Rad, Hercules, CA, USA). Sodium dodecyl sulfate-polyacrylamide gel electrophoresis $(8 \%)$ was performed to separate proteins, which were transferred to polyvinylidene difluoride membranes (Merck Millipore, Burlington, MA, USA). After soaking in blocking buffer ( $5 \%$ nonfat dry milk in Tris-buffered saline containing Tween-20 (TBS-T)) for $1 \mathrm{~h}$, membranes were incubated with primary antibodies specific for AhR (mouse monoclonal, Santa Cruz Biotechnology, Santa Cruz, CA, USA, \#SC-133088), HO1 (ADI-SPA-895, rabbit polyclonal, Enzo Stressgen, Farmingdale, NY, USA), SOD2 (ab13533, rabbit Immunoglobulin G (IgG), Abcam, Cambridge, United Kingdom), caspase 3 (rabbit polyclonal, Cell Signaling Technology, Danvers, MA, USA, \#9662S), and $\beta$-actin (mouse monoclonal $\mathrm{IgG}_{1}$, Santa Cruz, Santa Cruz, CA, USA, \#sc47778). Then, secondary antibodies (an anti-rabbit IgG horse radish peroxidase (HRP)-conjugated antibody 
(Cell Signaling Technology, \#7074S) and a goat antimouse IgG heavy and light chain (H\&L) HRP antibody (Abcam, \#ab97023)) were applied. Immunoreactions were detected with an enhanced chemiluminescence kit (Bio-Rad, Hercules, CA, USA). Protein expression levels were estimated using ImageJ gel analysis software (National Institutes of Health, Bethesda, MD, USA) and compared with the expression level of beta-actin.

\subsection{Statistical Analysis}

ABRTs were compared among groups using repeated measures ANOVA with Tukey's posthoc test for both pretreatment and post-treatment and for frequencies of $4,8,16$, and $32 \mathrm{kHz}$. mRNA or protein expression levels were compared among groups using ANOVA with Tukey's posthoc test. The values in the graphs are the means \pm standard deviations (SDs). All analyses were conducted using SPSS software (ver. 21.0; IBM Corp., Armonk, NY, USA). Statistical significance was defined as a $p$-value of less than 0.05 .

\section{Conclusions}

The present results establish the potential of $\mathrm{ZO}$ to attenuate CXP-induced auditory threshold shifts and inflammatory and apoptotic changes in cochleae. Because oxidative stress, inflammation, and apoptosis are shared mechanisms underlying ototoxicity caused by noise, aging, and aminoglycosides, $\mathrm{ZO}$ could be applied for various causes of ototoxic injuries. However, this study did not differentiate the mitochondrial expression levels of oxidative stress-related molecules from the whole-cell expression levels. In addition, clinical studies, including a toxicity analysis, should be performed before clinical use of $\mathrm{ZO}$.

Author Contributions: Conceptualization, S.Y.K. Investigation, C.H.L., D.-h.L., S.M.L, and S.Y.K. Methodology, S.Y.K. and D.-h.L. Writing—original draft, C.H.L. and S.Y.K. Writing-review and editing, C.H.L., D.-h.L., S.M.L., and S.Y.K. All authors have read and agreed to the published version of the manuscript.

Funding: This research was supported by funding from the National Research Foundation (NRF) of Korea (NRF-2016M3A9E8941669 and 2020R1A2C4002594).

Conflicts of Interest: The authors declare no conflicts of interest. The funders had no role in the design of the study; in the collection, analyses, or interpretation of data; in the writing of the manuscript; or in the decision to publish the results.

\section{References}

1. Langer, T.; am Zehnhoff-Dinnesen, A.; Radtke, S.; Meitert, J.; Zolk, O. Understanding platinum-induced ototoxicity. Trends Pharmacol. Sci. 2013, 34, 458-469. [CrossRef]

2. Garcia-Alcantara, F.; Murillo-Cuesta, S.; Pulido, S.; Bermudez-Munoz, J.M.; Martinez-Vega, R.; Milo, M.; Varela-Nieto, I.; Rivera, T. The expression of oxidative stress response genes is modulated by a combination of resveratrol and N-acetylcysteine to ameliorate ototoxicity in the rat cochlea. Hear. Res. 2018, 358, 10-21. [CrossRef]

3. Fetoni, A.R.; De Bartolo, P.; Eramo, S.L.; Rolesi, R.; Paciello, F.; Bergamini, C.; Fato, R.; Paludetti, G.; Petrosini, L.; Troiani, D. Noise-induced hearing loss (NIHL) as a target of oxidative stress-mediated damage: Cochlear and cortical responses after an increase in antioxidant defense. J. Neurosci. 2013, 33, 4011-4023. [CrossRef]

4. Watanabe, K.; Inai, S.; Jinnouchi, K.; Bada, S.; Hess, A.; Michel, O.; Yagi, T. Nuclear-factor kappa B (NF-kappa B)-inducible nitric oxide synthase (iNOS/NOS II) pathway damages the stria vascularis in cisplatin-treated mice. Anticancer Res. 2002, 22, 4081-4085.

5. Sheth, S.; Mukherjea, D.; Rybak, L.P.; Ramkumar, V. Mechanisms of Cisplatin-Induced Ototoxicity and Otoprotection. Front. Cell. Neurosci 2017, 11, 338. [CrossRef]

6. Callejo, A.; Sedo-Cabezon, L.; Juan, I.D.; Llorens, J. Cisplatin-Induced Ototoxicity: Effects, Mechanisms and Protection Strategies. Toxics 2015, 3, 268-293. [CrossRef] 
7. So, H.; Kim, H.; Lee, J.H.; Park, C.; Kim, Y.; Kim, E.; Kim, J.K.; Yun, K.J.; Lee, K.M.; Lee, H.Y.; et al. Cisplatin cytotoxicity of auditory cells requires secretions of proinflammatory cytokines via activation of ERK and NF-kappaB. J. Assoc. Res. Otolaryngol. 2007, 8, 338-355. [CrossRef]

8. Yang, Q.; Sun, G.; Yin, H.; Li, H.; Cao, Z.; Wang, J.; Zhou, M.; Wang, H.; Li, J. PINK1 Protects Auditory Hair Cells and Spiral Ganglion Neurons from Cisplatin-induced Ototoxicity via Inducing Autophagy and Inhibiting JNK Signaling Pathway. Free Radic. Biol. Med. 2018, 120, 342-355. [CrossRef]

9. Anniko, M.; Sobin, A. Cisplatin: Evaluation of its ototoxic potential. Am. J. Otolaryngol. 1986, 7, $276-293$. [CrossRef]

10. Van Ruijven, M.W.; de Groot, J.C.; Klis, S.F.; Smoorenburg, G.F. The cochlear targets of cisplatin: An electrophysiological and morphological time-sequence study. Hear. Res. 2005, 205, 241-248. [CrossRef]

11. Hartmann, J.T.; Lipp, H.P. Toxicity of platinum compounds. Expert Opin. Pharmacother. 2003, 4, 889-901. [CrossRef]

12. Kim, S.J.; Park, C.; Lee, J.N.; Park, R. Protective roles of fenofibrate against cisplatin-induced ototoxicity by the rescue of peroxisomal and mitochondrial dysfunction. Toxicol. Appl. Pharmacol. 2018, 353, 43-54. [CrossRef]

13. Youn, C.K.; Kim, J.; Jo, E.R.; Oh, J.; Do, N.Y.; Cho, S.I. Protective Effect of Tempol against Cisplatin-Induced Ototoxicity. Int. J. Mol. Sci. 2016, 17, 1931. [CrossRef]

14. Cobanoglu, H.B.; Vuralkan, E.; Arslan, A.; Mirasoglu, B.; Toklu, A.S. Is Hyperbaric Oxygen Therapy Effective in Cisplatin-Induced Ototoxicity in Rats? Clin. Exp. Otorhinolaryngol. 2019, 12, 66-71. [CrossRef]

15. Kandemir, F.M.; Yildirim, S.; Caglayan, C.; Kucukler, S.; Eser, G. Protective effects of zingerone on cisplatin-induced nephrotoxicity in female rats. Environ. Sci. Pollut. Res. Int. 2019, 26, 22562-22574. [CrossRef]

16. Wu, J.; Duan, Y.; Cui, J.; Dong, Y.; Li, H.; Wang, M.; Fan, S.; Li, D.; Li, Y. Protective effects of zingerone derivate on ionizing radiation-induced intestinal injury. J. Radiat. Res. 2019, 60, 740-746. [CrossRef]

17. Lee, W.; Hwang, M.H.; Lee, Y.; Bae, J.S. Protective effects of zingerone on lipopolysaccharide-induced hepatic failure through the modulation of inflammatory pathways. Chem. Biol. Interact. 2018, 281, 106-110. [CrossRef]

18. Lee, B.S.; Lee, C.; Yang, S.; Ku, S.K.; Bae, J.S. Renal protective effects of zingerone in a mouse model of sepsis. BMB Rep. 2019, 52, 271-276. [CrossRef]

19. Alibakhshi, T.; Khodayar, M.J.; Khorsandi, L.; Rashno, M.; Zeidooni, L. Protective effects of zingerone on oxidative stress and inflammation in cisplatin-induced rat nephrotoxicity. Biomed. Pharmacother. 2018, 105, 225-232. [CrossRef]

20. Mohamed, H.E.; Badawy, M.M.M. Modulatory effect of zingerone against cisplatin or gamma-irradiation induced hepatotoxicity by molecular targeting regulation. Appl. Radiat. Isot. 2019, 154, 108891. [CrossRef]

21. Soliman, A.F.; Anees, L.M.; Ibrahim, D.M. Cardioprotective effect of zingerone against oxidative stress, inflammation, and apoptosis induced by cisplatin or gamma radiation in rats. Naunyn Schmiedeberg's Arch. Pharmacol. 2018, 391, 819-832. [CrossRef] [PubMed]

22. Kaygusuzoglu, E.; Caglayan, C.; Kandemir, F.M.; Yildirim, S.; Kucukler, S.; Kilinc, M.A.; Saglam, Y.S. Zingerone ameliorates cisplatin-induced ovarian and uterine toxicity via suppression of sex hormone imbalances, oxidative stress, inflammation and apoptosis in female wistar rats. Biomed. Pharmacother. 2018, 102, 517-530. [CrossRef] [PubMed]

23. Karasawa, T.; Steyger, P.S. An integrated view of cisplatin-induced nephrotoxicity and ototoxicity. Toxicol. Lett. 2015, 237, 219-227. [CrossRef]

24. Sonawane, V.R.; Siddique, M.U.M.; Gatchie, L.; Williams, I.S.; Bharate, S.B.; Jayaprakash, V.; Sinha, B.N.; Chaudhuri, B. CYP enzymes, expressed within live human suspension cells, are superior to widely-used microsomal enzymes in identifying potent CYP1A1/CYP1B1 inhibitors: Identification of quinazolinones as CYP1A1/CYP1B1 inhibitors that efficiently reverse B[a]P toxicity and cisplatin resistance. Eur. J. Pharm. Sci. 2019, 131, 177-194.

25. Soobrattee, M.A.; Neergheen, V.S.; Luximon-Ramma, A.; Aruoma, O.I.; Bahorun, T. Phenolics as potential antioxidant therapeutic agents: Mechanism and actions. Mutat. Res. 2005, 579, 200-213. [CrossRef]

26. Aktan, F. iNOS-mediated nitric oxide production and its regulation. Life Sci. 2004, 75, 639-653. [CrossRef]

27. Ghosh, S.; May, M.J.; Kopp, E.B. NF-kappa B and Rel proteins: Evolutionarily conserved mediators of immune responses. Annu. Rev. Immunol. 1998, 16, 225-260. [CrossRef] [PubMed] 
28. Bennouna, S.; Denkers, E.Y. Microbial antigen triggers rapid mobilization of TNF-alpha to the surface of mouse neutrophils transforming them into inducers of high-level dendritic cell TNF-alpha production. J. Immunol. 2005, 174, 4845-4851. [CrossRef] [PubMed]

29. Gibbs, B.F.; Wierecky, J.; Welker, P.; Henz, B.M.; Wolff, H.H.; Grabbe, J. Human skin mast cells rapidly release preformed and newly generated TNF-alpha and IL-8 following stimulation with anti-IgE and other secretagogues. Exp. Dermatol. 2001, 10, 312-320. [CrossRef]

30. Dempsey, P.W.; Doyle, S.E.; He, J.Q.; Cheng, G. The signaling adaptors and pathways activated by TNF superfamily. Cytokine Growth Factor Rev. 2003, 14, 193-209. [CrossRef]

31. Yang, C.; Kaushal, V.; Shah, S.V.; Kaushal, G.P. Autophagy is associated with apoptosis in cisplatin injury to renal tubular epithelial cells. Am. J. Physiol. Renal. Physiol. 2008, 294, F777-F787. [CrossRef] [PubMed]

32. Mukherjea, D.; Jajoo, S.; Whitworth, C.; Bunch, J.R.; Turner, J.G.; Rybak, L.P.; Ramkumar, V. Short interfering RNA against transient receptor potential vanilloid 1 attenuates cisplatin-induced hearing loss in the rat. J. Neurosci. 2008, 28, 13056-13065. [CrossRef] [PubMed]

33. Kim, S.Y.; Jung, G.; Shim, Y.J.; Koo, J.W. The Novel Peptide Vaccine GV1001 Protects Hearing in a Kanamycin-induced Ototoxicity Mouse Model. Otol. Neurotol. 2018, 39, e731-e737. [CrossRef]

34. Chang, A.; Li, C.; Huang, J.; Pan, W.; Tian, Y.; Tang, J. Auditory Brainstem Response and Outer Hair Cell Whole-cell Patch Clamp Recording in Postnatal Rats. J. Vis. Exp. 2018, 135, e56678. [CrossRef]

35. Manji, S.S.; Miller, K.A.; Williams, L.H.; Dahl, H.H. Identification of three novel hearing loss mouse strains with mutations in the Tmc1 gene. Am. J. Pathol. 2012, 180, 1560-1569. [CrossRef]

36. Lee, C.H.; Park, S.S.; Lee, D.H.; Lee, S.M.; Kim, M.Y.; Choi, B.Y.; Kim, S.Y. Tauroursodeoxycholic acid attenuates cisplatin-induced hearing loss in rats. Neurosci. Lett. 2020, 722, 134838. [CrossRef]

37. Kim, S.Y.; Kim, J.K.; Park, S.H.; Kim, B.G.; Jang, A.S.; Oh, S.H.; Lee, J.H.; Suh, M.W.; Park, M.K. Effects of inhaled particulate matter on the central nervous system in mice. Neurotoxicology 2018, 67, 169-177. [CrossRef]

(C) 2020 by the authors. Licensee MDPI, Basel, Switzerland. This article is an open access article distributed under the terms and conditions of the Creative Commons Attribution (CC BY) license (http://creativecommons.org/licenses/by/4.0/). 